\title{
Estrategias de enseñanza-aprendizaje a través de videoconferencias
}

Pedro Luis Huergo-Tobar

Magíster en Educación. Universidad Cooperativa de Colombia, Neiva,

Colombia.

Correo electrónico:

pedro.huergo@campusucc.edu.co

Recibido: 31 de octubre del 2014

Aprobado: 30 de junio del 2015

Cómo citar este artículo: Huergo-Tobar, Pedro Luis. "Estrategias de enseñanzaaprendizaje a través de videoconferencias". Rastros Rostros 17.31 (2015): 65-77. Impreso. doi: http://dx.doi.org/10.16925/ ra.v17i31.1096

\section{Resumen}

Introducción: el presente artículo de investigación indaga acerca de la percepción que tienen los estudiantes y profesores de la Facultad de Ciencias Contables, Económicas y Administrativas (FCCEA) de la Universidad Cooperativa de Colombia (UCC), sede Neiva, sobre las estrategias de enseñanza-aprendizaje aplicadas a los acompañamientos pedagógicos. Metodología: la investigación es de enfoque cualitativo con apoyo cuantitativo. Resultados: se identifican las estrategias de enseñanza-aprendizaje que usan los profesores y, con base en los resultados, se proponen estrategias mediadas por la videoconferencia. Conclusiones: si bien se da importancia a las tutorías, los estudiantes no asisten a ellas y prefieren que se efectúen mediante la videoconferencia.

Palabras clave: acompañamiento pedagógico, estrategias de enseñanzaaprendizaje, EVAD, MICEA, tutorías, videoconferencia. 


\title{
Teaching-learning strategies through videoconferencing
}

\begin{abstract}
Introduction: this research article examines the perception of students and faculty at the School of Accounting, Economics and Management Sciences (FCCEA), Universidad Cooperativa de Colombia (UCC) in Neiva, based on teaching-learning strategies applied to pedagogical support. Methodology: this research has a qualitative approach with quantitative support. Results: the teaching-learning strategies used by teachers are identified and, based on results, videoconferencing mediated strategies are proposed. Conclusions: although importance is given to tutorials, students do not attend them and prefer that they are carried out by videoconference.
\end{abstract}

Keywords: teaching support, teaching-learning strategies, EVAD, MICEA, tutorials, videoconferencing.

\section{Estratégias de ensino-aprendizado através de videoconferências}

\section{Resumo}

Introdução: o presente artigo de investigação trata da percepção que tem os estudantes e professores da Faculdade de Ciências Contábeis, Económicas e Administrativas (FCCEA) da Universidade Cooperativa de Colômbia (UCC), na sede de Neiva, sobre as estratégias de ensino-aprendizado aplicadas aos acompanhamentos pedagógicos. Metodologia: a investigação é de enfoque qualitativo com apoio quantitativo. Resultados: identificam-se as estratégias de ensino-aprendizado usadas pelos professores e, baseado nos resultados, propõem-se estratégias mediadas pela videoconferência. Conclusões: embora se dá importância às tutorias, os estudantes não assistem a elas e preferem que sejam feitas através de videoconferência.

Palavras-chave: acompanhamento pedagógico, estratégias de ensinoaprendizado, EVAD, MICEA, tutorias, videoconferência. 


\section{Introducción}

El fenómeno abordado en el presente estudio es la poca participación de estudiantes en los acompañamientos pedagógicos. A pesar de que las coordinaciones académicas programan en el "Plan de trabajo semestral" (PTS) de los profesores un tiempo para el acompañamiento, la asistencia estudiantil a estos espacios es muy baja. Los estudiantes, al no tener la suficiente claridad sobre las actividades a realizar, no presentan los trabajos académicos a tiempo, lo que conlleva a solicitudes constantes para entrega de trabajos extemporáneos y traumatismos en los cierres de los cortes académicos.

La consecuencia previsible de seguir esta situación es la pérdida de un espacio de apoyo a la formación profesional, así como el desarrollo de las competencias que se desean fomentar en cada asignatura.

Buscar maneras novedosas a fin de hacer frente a la problemática crea la necesidad de efectuar una investigación que conduzca a hacer propuestas que fortalezcan estos procesos formativos. Con los avances tecnológicos, Internet y la videoconferencia se presentan como alternativas útiles para apoyar las actividades del aula de clase. Autores como Boniffil, Dougimas y Martínez et al., plantean en sus trabajos la necesidad de cambiar paradigmas en la manera de enseñar y aprender, de manera que el empleo de las tecnologías de la información y la comunicación (TIC), y en especial la videoconferencia, se presentan como herramientas apropiadas para romper las barreras que impiden los encuentros cara a cara, así como permiten efectuar una comunicación sincrónica ágil, clara y de calidad. Por lo tanto, este artículo propone realizar estos acompañamientos pedagógicos mediados por la videoconferencia como apoyo a las asignaturas en la modalidad presencial.

La experiencia sobre el particular en la UCC, sede Neiva, es nula. Se parte del supuesto según el cual los docentes de tiempo completo, medio tiempo y catedráticos, a pesar de manejar estrategias de enseñanza-aprendizaje para sus cátedras, no están preparados para el manejo de las TIC - en especial, la videoconferencia $-y$, por lo tanto, no poseen la fundamentación que les permita aplicar las teorías pedagógicas que habitualmente se usan en estos ambientes. Las horas de trabajo independiente requieren de un acompañamiento, el cual podría efectuarse mediado por la videoconferencia.

El compromiso de coordinadores y docentes involucrados es realizar acciones encaminadas a afrontar de manera exitosa todas estas previsibles situaciones. Esto supone efectuar investigaciones dirigidas a elaborar propuestas frente al proceso de uso, a los nuevos aprendizajes, a la aplicación de teorías pedagógicas y de las tecnologías requeridas para el proceso de formación de profesionales con pertinencia regional, todo lo cual brinde una prospectiva positiva al interior de la UCC. Los docentes que adquieran experiencia en la aplicación de estas herramientas de acompañamiento en los procesos de aprehensión cognitiva, dan seguridad a todas las partes involucradas y son firmes candidatos para promover el cambio $y$, por ende, comprometerse con su implementación.

Por otro lado, actualmente existen varias opciones de software libre para implementar la videoconferencia en los computadores de uso personal sin mayores exigencias de tipo técnico, lo cual permite que esta opción esté al alcance de todos.

De lo anteriormente expuesto, surge la pregunta: ¿qué estrategias de enseñanza-aprendizaje se pueden implementar en los acompañamientos pedagógicos a los estudiantes de la Facultad de Ciencias Contables, Económicas y Administrativas de la UCC, sede Neiva, a través de la videoconferencia?

\section{Antecedentes}

De acuerdo con los lineamientos del presente estudio, se analiza lo que ha sido el uso de la videoconferencia y las aulas virtuales en el área educativa.

La investigación efectuada por Dougiamas sobre las experiencias de aprendizaje de los estudiantes y docentes en el curso de posgrado a través de Internet, denominado "constructivismo", muestra la relación entre las experiencias de los participantes y el website. Se concluye que las interacciones online entre los estudiantes constituyen un diálogo débil; las razones atribuidas son presión y falta de tiempo. Las estrategias a futuro son un cambio radical en la estructura de las videoconferencias.

El trabajo efectuado por Martínez et al. informa sobre los retos de innovación para los docentes con el establecimiento de nuevos grados en el marco del Espacio Europeo de Educación Superior. Se considera allí que el desarrollo de conocimientos transversales implica un cambio en el paradigma tradicional de la educación universitaria, ya que se pasa del protagonismo del profesor a la participación activa del alumno en su proceso de enseñanza-aprendizaje. Para este cambio se debe contar con métodos pedagógicos adecuados, los cuales respondan a las nuevas necesidades 
de los alumnos frente a los nuevos y rápidos cambios que supone esta adaptación. En este trabajo se muestran los instrumentos de la plataforma MOODLE utilizados en la Facultad de Ciencias Empresariales de la Universidad Complutense de Madrid.

Sobre la evaluación pedagógica de cursos virtuales, Pérez muestra que la problemática se centra en la decepción y pérdida de credibilidad de los cursos de enseñanza mediados por TIC, esto en razón a que estas relegan lo pedagógico a un segundo plano, primando el desarrollo y venta de plataformas que no están basadas en modelos psicopedagógicos del conocimiento. Las conclusiones son preocupantes: al existir carencias de una metodología propia de la formación telemática, la consecuencia es que las técnicas se encuentren alejadas del objeto, con escasos elementos significativos de aprendizaje, de manera que se favorece la observación y la repetición pasiva.

Con el propósito de realizar una mejor gestión del mejoramiento permanente de la calidad de la educación virtual a distancia, es preciso unir de manera óptima la educación virtual con la no virtual, evitando contraponer modalidades educativas. Se requiere con respecto a la calidad de la educación, en especial de la virtual a distancia (EVAD), del papel de la innovación tecnológica, del cambio de paradigmas de enseñanza y aprendizaje en la evaluación y gestión de la calidad. Al articular las distintas modalidades se examinan propuestas y estrategias para la evaluación de la EVAD como base de efectiva gestión, con el fin de asegurar, mantener y mejorar continuamente su calidad (Silvio). Sobre esto, Uribe (2007) estima necesario lograr el desarrollo y aplicación de nuevas tecnologías educativas y de información, centradas en la adquisición de estas competencias en los estudiantes logrando mayor eficiencia y eficacia en los procesos académicos y administrativos, brindando mejores oportunidades de aprendizaje, e incorporarlas al desarrollo curricular, además de ofrecer enseñanza semipresencial y virtual como mecanismo para aumentar la cobertura, tener presencia regional, absorbiendo las nuevas Tic.

De acuerdo con lo que plantea Bonfill sobre los factores críticos del empleo de la videoconferencia en un sistema de educación a distancia en las aulas virtuales en una universidad, los tutores cuando la utilizan muestran sus temores, limitaciones, cambios y progresos en su uso. Además, presenta la videoconferencia en la educación virtual como herramienta de interacción entre profesor y alumno, gracias a la cual - sin antecedente habitual de trabajo y sin costo-, pueden disfrutar de expositores de primera línea, con temas y presentaciones actualizados de alto nivel académico. Por otra parte, Linares et. al enfatizan en la estructura metodológica y el papel del facilitador en las diferentes formas organizativas del proceso docente dentro del sistema de clases mediadas por la videoconferencia, demostrandola importancia dela sistematización de la enseñanza y la forma en que se organizan los contenidos desde un nivel de menor a otro de mayor complejidad. Además concluye que esta herramienta es útil para estudiantes que — dada la distancia - no pueden acceder de manera frecuente a las asesorías metodológicas presenciales. Asimismo, Ardila afirma que la incorporación de las tecnologías de información y comunicación en la educación obliga a replantear la práctica docente para dar respuesta a cuáles son las competencias esperadas y las funciones que orientan la labor de enseñanza-aprendizaje. De lo anterior se infiere que aquellos trabajos efectuados desde la virtualidad requieren, para su éxito o fracaso, de una planeación y dedicación minuciosa en su desarrollo. Se presenta así la videoconferencia como herramienta idónea para dar solución al problema de movilidad del estudiante, permitiéndoles conocer los contenidos de la asignatura de una manera interactiva, de manera que los estudiantes experimentan una nueva forma de adquirir los conocimientos y satisfacen las dudas sobre determinados temas inmersos en la aplicación de este modelo pedagógico.

\section{Marco teórico}

Las categorías con las que se desarrolló el marco teórico se toman como base desde lo que plantea De Gregori (99), citado y descrito por Velandia (Metodología 39), sobre el ciclo cibernético de transformación (ССT). Con esto se buscan articular los referentes teóricos seleccionados. Se afirma que el primer reto es ayudarle al estudiante en el conocimiento de su estructura y del grado de madurez de sus áreas cerebrales. Se presenta autoconducción del estudiante cuando este organiza la relación entre sus tres procesos mentales (saber, izquierdo; crear, derecho, y hacer, central).

Según De Gregori, el cerebro es tri-uno (teoría tricerebral), y la denominación que le asigna a esos tres bloques son: cerebro central o proceso operativo factual; el cerebro derecho emocional o proceso intuitivo, y la parte izquierda lógica o proceso analítico del cerebro. Esto es, si el cerebro tri-uno es estimulado, 
en la medida en que el docente logre identificar en sus alumnos las áreas predominantes de su cerebro, podrá efectuar la estimulación correcta en sus procesos de aprendizaje mediante el acompañamiento pedagógico a través de la videoconferencia.

Solano refiere la videoconferencia como un sistema multimedia audiovisual e interactivo, bidireccional o unidireccional, sincrónico o asincrónico, el cual permite la transmisión y recepción de información visual y auditiva de manera flexible, con grandes posibilidades didácticas. Supera las limitaciones espacio-temporales y la orientación textual de otras herramientas en las que se pueden desarrollar sistemas mixtos de enseñanza (b-learning) y teleenseñanza, logrando así aumentar la calidad en la educación con la incorporación de recursos externos aplicables en todos los niveles de enseñanza y modalidades educativas. En esta interacción dinámica posibilitada por el empleo de la videoconferencia, se crea un ambiente propicio para la construcción del conocimiento.

Ese construccionismo, según Vygotsky, citado por Jáuregui, Canto y Ros, en el que "el aprendizaje se construye cuando los aprendientes participan en interacciones sociales tomando como punto de partida tareas que requieren colaboración" (749). Además, Donolo, Chiecher y Rinaudo (6) afirman que el contexto de la educación se debe analizar bajo determinadas circunstancias y en ciertas condiciones, de manera que las interacciones puedan tener un impacto positivo sobre la construcción de conocimientos que realiza el alumno sean estos ambientes virtuales o presenciales. El upayador o coaching, propuesto por De Gregori, que en este caso es el profesor, es el encargado de efectuar el acompañamiento pedagógico a través de la estrategia centrada en la tutoría (Velandia, Modelo pedagógico 91).

De acuerdo con FONDEP (5), el acompañamiento pedagógico se define como asesoría especializada brindada de manera planificada, continua, contextualizada, interactiva y respetuosa, encaminado a enriquecer la calidad de los aprendizajes de los educandos, del ejercicio docente como líderes innovadores y de la gestión escolar. Para el Ministerio de Educación de Perú (MED), el acompañamiento pedagógico es el recurso pedagógico basado en el intercambio de experiencias entre docente y educando, sin distinción de niveles de superioridad y jerarquía; requiere una interacción auténtica que cree relaciones horizontales, en un ambiente de aprendizaje y de intervención pedagógica pertinente al entorno de la institución, de modo tal que permita un proceso de intercambio profesional producido a través del diálogo, y a partir de la observación y evaluación del trabajo en el aula. A fin de lograr esto, es necesario tener la capacidad de compartir y la disposición para establecer compromisos enfocados en el crecimiento mutuo.

Ahora bien, dado que este proceso se realiza en ambientes virtuales, resulta pertinente consultar sobre el conectivismo; sobre esto Leal-Fonseca, precisa la necesidad de identificar conexiones e incluir la tecnología, ubicando las teorías de aprendizaje en la actualidad. De acuerdo con Siemens, el conectivismo es una teoría de aprendizaje para la era digital. Es la integración de principios estudiados por teorías del caos, redes, complejidad y autoorganización. Sobre el proceso de aprender y conocer, el conectivismo lo plantea como una formación basada en redes de nodos, o fuentes de información, especializadas e interconectadas. Asimismo, el conocimiento puede residir en dispositivos, y la tecnología da viabilidad al aprendizaje eficaz y preciso para acceder al conocimiento contemporáneo.

Este desarrollo de enseñanza-aprendizaje dado en la virtualidad requiere de estrategias para su efectividad. En este sentido, Nisbel, citado por Meoño, afirma que las estrategias de aprendizaje son métodos que sirven de base a la realización de las tareas intelectuales, conductas y tareas que facilitan las destrezas de aprendizaje. Además, Naranjo, Uribe y Valencia de Veizaga aseveran que entre las estrategias de aprendizaje más pertinentes en la educación virtual se encuentran la lectura, el análisis y la solución de problemas, la realización de tareas, la consulta de bases de datos, las lecciones electrónicas, las preguntas a expertos, la interacción con el tutor, así como el acceso a diferentes materiales de aprendizaje en foros, chats, construcción de micromundos y aulas virtuales.

Por otro lado, Díaz y Hernández definen las estrategias de enseñanza-aprendizaje como procedimientos o recursos utilizados con el fin de promover aprendizajes significativos. Entre las estrategias se encuentran el diseño y empleo de objetivos e intenciones de enseñanza, preguntas insertadas, ilustraciones, modos de respuesta, organizadores anticipados, redes semánticas y mapas conceptuales.

Sobre esto, Díaz y Hernández plantean cómo los ítems que conforman las estrategias de aprendizaje son la búsqueda de alternativas novedosas para la selección, organización y distribución del 
conocimiento, asociadas al diseño y promoción de estrategias de aprendizaje e instrucción cognitiva. Resalta también la importancia de promover con el manejo del grupo la interacción entre el docente y sus alumnos, así como entre los alumnos mismos, mediante el empleo de estrategias de aprendizaje cooperativo. Además enfatiza en las teorías que se deben tener presentes para la creación de estrategias de aprendizaje: la teoría del aprendizaje verbal significativo, la teoría sociocultural del desarrollo y del aprendizaje y las teorías del desarrollo humano de la información que aportan a la concepción constructivista de la enseñanza y del aprendizaje, así como enfatizan sobre la naturaleza social y la función socializadora de la educación escolar, la educación escolar misma y los procesos de socialización y de construcción de la identidad personal.

Las anteriores teorías proporcionan la base para implementar la matriz que se propone a continuación, y que además sirve como fundamento de esta investigación. Ver figura 1.

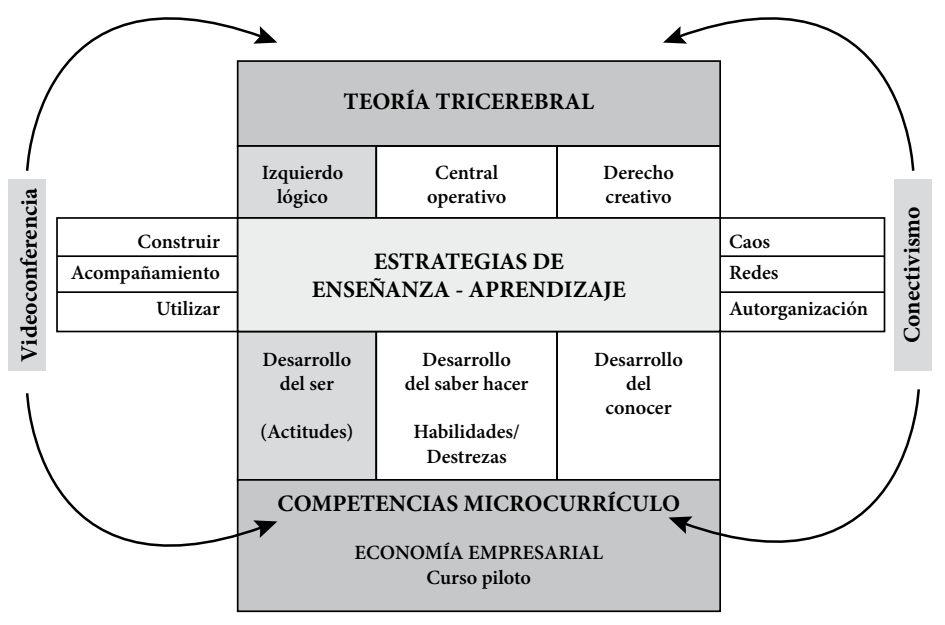

Figura 1. Matriz categorías estrategias de enseñanza-aprendizaje.

Fuente: elaboración propia a partir de información recopilada en De Gregori, W. Construcción Familiar-Escolar de los tres cerebros. Bogotá: Kimpres Ltda., 2002. Impreso. Meoño, Jorge. "IV Taller pedagógico, Estrategias de enseñanza aprendizaje, Institución Educativa 'Juan Manuel Itiguerri”. Slideshare. 2008. Web. Presentación; Naranjo, Edilma, Alejandro Uribe \& Martha Valencia De Veizaga. "La educación virtual y su aceptación en la Escuela Interamericana de Bibliotecología de la Universidad de Antioquia”. Revista Interamericana de Bibliotecología 29.2 (2006): 13-42. Impreso. Solano, Isabel. Las TIC como recursos en los procesos de comunicación didáctica. Tema 5: estrategias metodológicas. Digitum, 2010. Presentación; Siemens, G. (2005) “Connectivism: A Learning Theory for the Digital Age”. International Journal of Instructional Technology \& Distance Learning 2.1 (2005): 1-8. Web.http://er.dut.ac.za/bitstream/handle/123456789/69/Siemens_2005_Connectivism_A_learning_theory_for_the_digital_age.pdf?sequence=1\&isAllowed=y Web. Agosto 8 de 2011.

\section{Enfoque metodológico}

Esta investigación tiene un enfoque cualitativo descriptivo e interpretativo, con apoyo cuantitativo. Se aplica una encuesta tipo Likert realizada a docentes y alumnos, de acuerdo con las escalas cuantitativas con el fin de medir actitudes de Likert y preguntas politómicas.

La información fue recopilada a partir del análisis de datos cualitativos obtenidos del instrumento elegido para la información, mediante el desarrollo de un grupo focal. Se usó un cuestionario no restringido o abierto, el cual se organizó en concordancia con las categorías establecidas. Las razones para la elección de este instrumento se expresan de manera acertada en el documento elaborado por Páramo:

El grupo focal (GF) produce un tipo particular de datos cualitativos produciendo datos focalizados o sea este instrumento se centra en estímulos o situaciones externas de interés del investigador y es relativamente organizado por un moderador. (149)

Se efectuó el grupo focal tanto a estudiantes (en la emisora de la UCC, sede Neiva), como a los docentes en la sala de profesores de la FCAEC. A fin de realizar un análisis acertado y riguroso, las respuestas de cada pregunta del cuestionario se interpretan con la 
elaboración de unidades hermenéuticas empleando el Software Atlas Ti versión 5.2, el cual es utilizado para análisis cualitativo de textos y, tal como lo describe Nieto, facilita el análisis e interpretación de los mismos, así como su selección, codificación, anotación, la comparación y construcción de relaciones. Muñoz (5) define los codes o códigos como la unidad básica de análisis y son comprendidas como conceptualizaciones, resúmenes o agrupaciones de citas provenientes de fragmentos de texto, imágenes o sonidos.

\section{Población y muestra}

La población comprende a los estudiantes y docentes del semestre в 2011 en cada programa de la facultad, los cuales fueron suministrados por el Departamento de Admisiones, Registro y Control de la UCC, sede Neiva': los estudiantes de los programas suman 194 a octubre 20 del 2011. Los coordinadores de los programas notifican que existen un total de 39 docentes en la facultad.

Para determinar los tamaños maestrales, se emplea el software libre Decisión Analyst stATs ${ }^{\mathrm{TM}}$ 2.0, partiendo de los siguientes parámetros: nivel de confianza deseado del 95\%; margen de error máximo admitido (aceptable) del 8\%, y nivel de porcentaje estimado de la muestra de $50 \%$. El programa arrojó como tamaño de muestra representativa para 85 encuestas a aplicar a estudiantes y 31 docentes. La encuesta aplicada se aprecia en el apéndice A.

Se escogen dos grupo focales (GF), entre docentes y estudiantes. Se les aplica el Test de Revelador Tríadico, expuesto por De Gregori, a los estudiantes del semestre B 2011, pertenecientes a la asignatura de "Economía Empresarial", a fin de conocer su área cerebral dominante e identificar qué áreas deben ser cultivadas.

\section{Resultados}

Se efectúa un paralelo de los resultados obtenidos de la encuesta aplicada entre docentes y estudiantes. De lo anterior se infiere como la gran mayoría de las percepciones entre estudiantes sobre las temáticas indagadas, resultan muy similares exceptuando algunas, tales como solicitar información de cómo se debe efectuar el trabajo asignado, el desarrollo del acompañamiento de manera planificada, continua y

Martha Stella Cadena Manjarres, directora Departamento Admisiones, Registro y Control, UCC, sede Neiva contextualizada y en la eficiencia de los acompañamientos (ver apéndice B).

Los resultados del Test de Revelador Triádico se observan en la figura 2. Se halló que el grupo es más operativo y descuidado tanto en el área lógica como en el área emocional, a fin de desarrollar de manera armónica los tres lados del cerebro, las estrategias se deben dirigir a cultivar las áreas en las que se presenta debilidad.

\begin{tabular}{|c|c|c|c|c|}
\hline & & \multicolumn{3}{|c|}{$\begin{array}{l}\text { REVELADOR TRIADICO } \\
\text { Economia empresarial } 8-2011\end{array}$} \\
\hline & & C. izquierdo - & - C. Central & - C. derecho \\
\hline $\begin{array}{l}\text { 4. Nivel de Comando } \\
\text { (Mentalización } \\
\text { Decision) }\end{array}$ & 4 & $\begin{array}{c}5+22=4 \\
\text { EPISTEMOLOGIA }\end{array}$ & $\begin{array}{c}13+19=4 \\
\text { ADMINISTRAC }\end{array}$ & $\begin{array}{r}3=4 \\
\text { MistiCA }\end{array}$ \\
\hline $\begin{array}{l}\text { 3. Nivel de asesoría } \\
\text { (Técnico } \\
\text { Informacional) }\end{array}$ & 3 & $\begin{array}{l}1+11+15=3 \\
\text { INVESTIGACION }\end{array}$ & $\begin{array}{c}21+23=4 \\
\text { PLANEACIÓN }\end{array}$ & $\begin{array}{c}6+24=3 \\
\text { PERCEPC. ALFA }\end{array}$ \\
\hline $\begin{array}{l}\text { 2. Nivel de } \\
\text { Animación (Mando } \\
\text { medios Transmisión) }\end{array}$ & 2 & $\begin{array}{c}12+16=3 \\
\text { CLASIFICACIO }\end{array}$ & $\begin{array}{l}2+25+26=4 \\
\text { PROFESIÓN }\end{array}$ & $\begin{array}{l}4+14+20=4 \\
\text { CREATIVDAD }\end{array}$ \\
\hline $\begin{array}{l}\text { 1. Nivel de } \\
\text { Ejecución } \\
\text { (Operacional) }\end{array}$ & 1 & 1 \begin{tabular}{c|}
$8+27=4$ \\
LENGUAJE
\end{tabular} & \begin{tabular}{|c|}
$17+18=3$ \\
UCHA DE SOBRE \\
MVEN/REPRODUC \\
\end{tabular} & $\begin{array}{l}7+9+10=3 \\
\text { C AFECTIVIDAD }\end{array}$ \\
\hline
\end{tabular}

Figura 2. Revelador triádico por niveles de actuación.

Fuente: elaboración propia a partir de De Gregori, W. Construcción Familiar-Escolar de los tres cerebros. Bogotá: Kimpres Ltda., 2002. Impreso.

Según la ley de proporcionalidad propuesta por De Gregori (2002), el docente debe tomar estrategias metodológicas que le permitan al estudiante adquirir o asimilar los conocimientos de una manera práctica (figura 3).
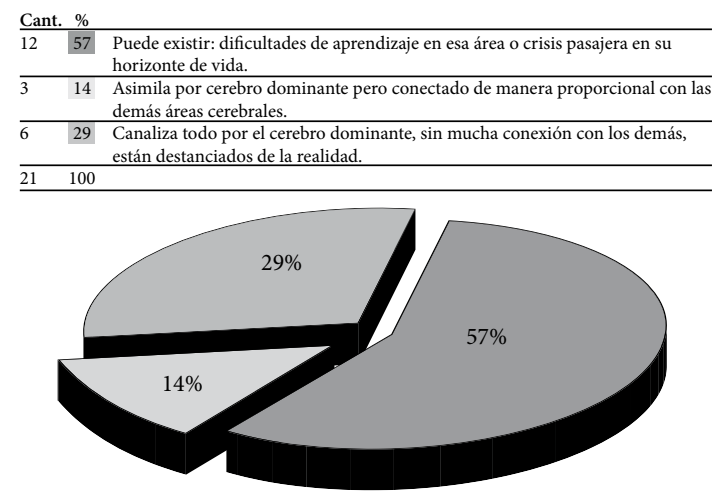

Figura 3. Diagnóstico por la ley de proporcionalidad en estudiantes de Economía Empresarial.

Fuente: elaboración propia a partir de De Gregori, W. Construcción Familiar-Escolar de los tres cerebros. Bogotá: Kimpres Ltda., 2002. Impreso. 
Los principales codes del árbol conceptual muestran cómo las estrategias de enseñanza-aprendizaje más empleadas en los docentes de la Facultad de Ciencias Contables, Económicas y Administrativas de la UCC, sede Neiva, son bastante variadas. Con relación a los profesores se destacan estrategias tales como la cátedra magistral, la investigación en el aula y el foro, así como los trabajos de aplicación, la asesoría a grupos de trabajo, el trabajo en equipo, hasta el desarrollo de talleres en clase y las lecturas que el docente previamente ha seleccionado, de modo que es posible afirmar que varios docentes emplean (Micea) Metodología Interdisciplinaria Centrada en Equipos de Aprendizaje (figura 4).

Con relación a los estudiantes, las consultas bibliográficas, el repasar apuntes, los trabajos en grupos, las exposiciones y el método de roles son las diferentes estrategias que afianzan el conocimiento impartido en clase (figura 5).

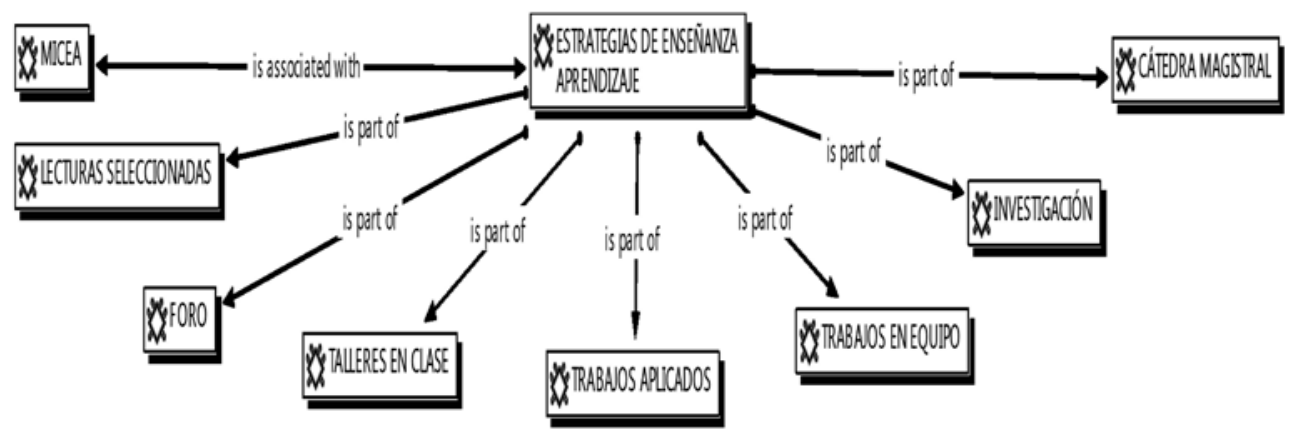

Figura 4. Estrategias de enseñanza-aprendizaje más empleadas por los profesores.

Fuente: elaboración propia.

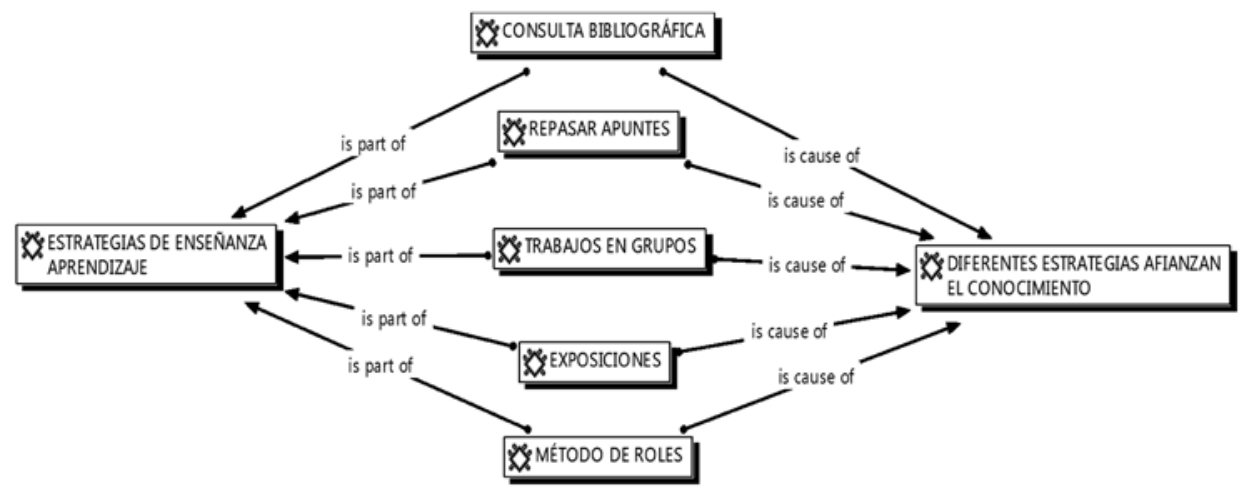

Figura 5. Estrategias de enseñanza-aprendizaje más empleadas por los estudiantes.

Fuente: elaboración propia.

Luego de la triangulación de los datos obtenidos, y si se toman como eje articulador las categorías establecidas para la presentación de los resultados del proceso investigativo, se proponen varias categorías. En la primera (constructivismo-acompañamiento pedagógico) del Cuestionario Likert, se obtiene una opinión aceptable de los profesores y del grupo focal. Ambas muestras poblacionales coinciden en la profundización que se logra en los temas. Se resalta que en la percepción de los profesores se obtiene con los acompañamientos pedagógicos una construcción de pensamiento crítico, y se mejora la capacidad de proponer soluciones alternas. Los estudiantes insisten sobre la necesidad de interacción entre el docente y el discente, de manera que se abran espacios para el fortalecimiento de la enseñanza y el aprendizaje (este debe ser un espacio donde se aclaren dudas). Los hallazgos encontrados están en concordancia 
con lo afirmado por Jáuregi, Canto y Ros; Vygotsky, citado por los autores anteriormente mencionados; y Rinaudo et al., quienes coinciden en afirmar que el interés por construir conocimiento en clase se manifiesta a través de la interacción docente-estudiante y en los acompañamientos pedagógicos; los resultados de esa construcción de conocimiento en la interacción efectuada, es más percibida en los docentes que en los estudiantes.

Con respecto a la segunda categoría (conectivismo, caos-redes-autoorganización), la información obtenida en el cuestionario muestra que la percepción en los docentes es aceptable, y en el grupo focal las opiniones son heterogéneas. Algunos lo ven viable, otros no tienen buen concepto de la herramienta, pero todos coinciden en que la clave del éxito es el uso guiado por el profesor. En el cuestionario también esta categoría es valorada positivamente por los estudiantes, y en el GC se presenta opinión homogénea sobre la viabilidad en el uso de la videoconferencia, conformación de redes y parcial opinión frente a la capacidad de seleccionar y dar buen tratamiento a la información obtenida de Internet. Afirman que se presenta mucho "copie y pegue" en los trabajos académicos. Por otra parte, ven de manera positiva el aporte al mejoramiento en la elaboración de exposiciones, pero advierten que en las consultas se debe ser cauto en su uso. Se coincide en que la clave del éxito está en los horarios y se recomienda se efectúen los fines de semana.

Cabe resaltar en la interpretación de los resultados que el deseo de acceder a nuevas tecnologías que faciliten el aprendizaje - tal como lo denomina Simmens -, en el caos, el construir y participar en redes y el acceder a conocimiento nuevo a través de la virtualidad, en general, se hace más palpable en los estudiantes que en los docentes.

En la tercera categoría (competencias del microcurrículo - ser-conocer-saber hacer-), la actitud en los docentes es aceptable y en el GF destacan la necesidad de la existencia de compromiso para el mutuo entendimiento de los logros. En los estudiantes esta categoría es percibida de manera aceptable. En el GF se llama la atención sobre el poco trabajo independiente y la preferencia por su desarrollo en clase, a fin de acceder a un oportuno acompañamiento. Según la Organización de las Naciones Unidas para la Educación la Ciencia y la Cultura (Unesco), el concepto de competencia es el pilar del desarrollo curricular y el incentivo tras el proceso de cambio; ese concepto puede emplearse como principio organizador del currículo. Estas competencias al ser triadizadas (competencias para el desarrollo del ser: actitudes - derecho-; competencias para el desarrollo del conocer -izquierdo-; y competencias para el desarrollo del saber hacer - central-), buscan estimular las distintas áreas del cerebro como lo afirma De Gregori.

Con respecto a la cuarta categoría (estrategias de enseñanza-aprendizaje), los docentes opinan que estas son el plan de trabajo, el monitoreo e interacción docente-discente constante, Micea, lecturas y talleres en clase. Por su parte, los estudiantes opinan que son las tutorías solución de inquietudes en clase, el repaso de apuntes y las exposiciones. Las estrategias de aprendizaje son los procedimientos o recursos utilizados por el agente de enseñanza con el fin de promover aprendizajes significativos. Sobre esto, Meoño, Velandia (Modelo pedagógico) y Nisbel coinciden en afirmar que las estrategias de enseñanza son procesos que sirven de base a la realización de las tareas asignadas a los estudiantes.

\section{Conclusiones}

Como respuesta al objetivo de la fase de investigación, las estrategias más empleadas en la FCCEA, sede Neiva, son: el trabajo en equipo, Micea, las lecturas efectuadas a consultas bibliográficas, talleres en clase, tutorías en la clase, el plan de trabajo, monitoreo e interacción docente-discente constante y exposiciones.

Complementando lo anterior, las estrategias de enseñanza aprendizaje para realizar acompañamiento pedagógico mediado por la videoconferencia a los estudiantes de la FCCEA, sede Neiva, se ubican en la investigación documental: son las cinco contempladas en la Metodología Micea de Velandia (Metodología): la estrategia y momento centrado en la propuesta del maestro, la estrategia y momento centrado en el estudiante, la estrategia y momento de los equipos de aprendizaje, la estrategia y momento de tutoría y asesoría y la estrategia y momento de socialización de experiencias y referenciación. Las anteriores se complementan con el empleo del diálogo socrático, según Acosta, y la estrategia de enseñar niveles de lectura, según Salas.

Es necesario en la propuesta pedagógica adaptar, combinar y contextualizar - de una manera planificada-, el conocimiento obtenido, a fin de ser desarrollado en la asignatura piloto. 
De los hallazgos obtenidos en la triangulación efectuada, se puede afirmar que los estudiantes perciben lo importante que resultan los acompañamientos pedagógicos, así como idóneas las estrategias allí desarrolladas, con positiva percepción en la construcción del conocimiento que allí se desarrolla. Sin embargo, no asisten a los acompañamientos, no los usan con una frecuencia acorde con la importancia por ellos expuesta.

Las principales barreras para asistir son los horarios en horas laborables o en horas que se cruzan con los horarios de clase; no todos los docentes poseen horas de acompañamiento asignadas y los estudiantes al recurrir a los que están presentes no reciben una orientación adecuada. El software para implementar este acompañamiento, en los docentes es el Skype, y en los estudiantes es el MSN Hotmail.

Finalmente, existe más favorabilidad para adoptar el cambio en los estudiantes que en los docentes; este es un reto que deben asumir en conjunto los docentes del programa para adaptar esta TIC y así dar solución a la problemática objeto de conocimiento.

\section{Referencias}

Acosta, Carlos. "Efectos del diálogo socrático sobre el pensamiento crítico en estudiantes universitarios". Psicología desde el Caribe. Universidad del Norte. No. 10: 1-26, 2002. Web. 5 Dic. de 2011. <http://www.redalyc. org/pdf/213/21301002.pdf>.

Ardila, Mireya. "Docencia en ambientes virtuales: nuevos roles y funciones, Universidad Pedagógica Nacional 2". Revista Virtual Universidad Católica del Norte 28. (2009): 1-15. Web. <http://scielo.sld.cu/scielo. php?pid=S1561-31942007000200015 \&script=sci_abstract>

Bonfill, Clara. "Clases virtuales a través de videoconferencias: Factores críticos vivenciados por los tutores en un sistema de educación a distancia”. Temas de Management. Edición especial sobre gestión del conocimiento. Vol 5: 12-20. Web. 5 Ene. 2012. <https:// www.ucema.edu.ar/cimei-base/download/research/ 45_Bonfill.pdf>

Campo, José, Mercado, Juan Mercado y Efraín Nocua. "Implementación de un prototipo de Aula Virtual en la Universidad de Santander, sede Bucaramanga". Universidad de Santander-Udes. Web. 5 Dic. 2011. $<$ http://www.colombiaaprende.edu.co/html/ mediateca/1607/articles-108379_archivo.pdf>
Castaño, Wilson y Alejandro Uribe. La formación en competencias tecnológicas e informacionales de futuros bibliotecólogos aprovechando la plataforma educativa Moodle: caso Escuela Interamericana de Bibliotecología de Antioquia 2007 -2008. Web. 20 de Junio de 2011. Conferencia.

De Gregori, Waldemar. Construcción familiar-escolar de los tres cerebros. Bogotá: Editorial Kimpres Ltda., 2002. Impreso.

Díaz, Frida y Hernández, Gerardo. Estrategias docentes para un aprendizaje significativo. Una interpretación constructivista. Segunda edición. México: McGraw-Hill, 2004. Web. 3 Feb. 2012. <https://jeffreydiaz.files. wordpress.com/2008/08/estrategias-docentes-para-un-aprendizaje-significativo.pdf>

Donolo, Danilo, Analía Chiecher y María Cristina Rinaudo. "Estudiantes en entornos tradicionales y a distancia". RED, Revista de Educación a Distancia 10. (2013). Web. 10 Feb. 2012. <http://www.um.es/ead/red/10/ chiecher.pdf>

Dougiamas, Martin y Peter Taylor. "Análisis interpretativo de la construcción de un curso a través de Internet usando una nueva herramienta de generación de cursos llamada Moodle". Curtin University of Technology. Perth. 2002. Web. 4 feb. 2012. <http://www.adelat. org/media/docum/moodle/docum/DocudeIgnacio. pdf>

FONDEP. Guía para el acompañamiento pedagógico de proyectos de innovación en la regiones. Fondo Nacional de Desarrollo de la Educación Peruana-FondeP, 2008: 5. Web: 11 Feb. 2012. <http://es.slideshare.net/perulina/guiadeacompanamiento $>$.

Jáuregui, K., Canto, S., y Ros, C. "La interculturalidad a través de la videoconferencia". Actas del XVI Congreso Internacional ASELE. Universidad de Utrecht. Centro Virtual Cervantes, 2005. Web. 6 Feb. 2012. $<$ http://cvc.cervantes.es/ensenanza/ biblioteca_ele/ asele/pdf/16/16_0747.pdf >

Leal-Fonseca, Diego E. "Conectivismo: Una teoría de aprendizaje para la era digital". Universiad de lo Andes. 2007. Web: Web. Febrero 7 de 2012. <http:// www.scribd.com/doc/201419/Conectivismo-una-teoria-del-aprendizaje-para-la-era-digital>

Linares Guerra, Maritza et al. "Guion de video conferencia para orientaciones metodológicas a los facilitadores de biología celular y molecular". Rev. Ciencias Médicas 1.2 (2007): 145-165. Web. 20 junio 2012. <http://scielo. sld.cu/ scielo.php?pid=S156131942007000200015\&script=sci_abstrac $>$ 
Martínez, Elena, Marta Fossas y Francisco Cobo. "De profesor a Community Manager: El potencial transformador de MOODLE en las relaciones docente-alumnos". RELADA-Revista Electrónica de ADA-Madrid, 4.2, 2010. 112-121. Web. http://polired.upm.es/index.php/relada/article/view/101/97

Meoño, Jorge. "Iv Taller pedagógico, Estrategias de enseñanza aprendizaje, Institución Educativa Juan Manuel Itiguerri. Slideshare. 2008. Web. http://www. slideshare.net/jmiturregui/estrategias-de-enseanza-aprendizaje-presentation. Presentación.

Ministerio de Educación de Perú (MED). “Acompañamiento pedagógico; lineamientos y estrategias generales para la supervisión pedagógica”, 2008. Web. 15 Dic. de 2011. <http://www.slideshare.net/walitron/acompaamiento-pedagogico-5639894>. Presentación.

Muñoz, Juan. “AtLAS.Ti Versión 3.0.” Universidad Autónoma de Barcelona, 2005. Web. <https://www.academia. edu/958909/An\%C3\%A1lisis_de_datos_textuales_ con_Atlas.ti_5>

Naranjo, Edilma, Alejandro Uribe y Martha Valencia de Veizaga. "La educación virtual y su aceptación en la Escuela Interamericana de Bibliotecología de la Universidad de Antioquia”. Revista Interamericana de Bibliotecología 29.2 (2006): 13-42. Impreso.

Nieto, Luz. Métodos y técnicas de investigación en las ciencias sociales (énfasis análisis cualitativo y computo). 2002. Web. 5 Dic. 2011 <http://ambiental.uaslp.mx/ docs/ LMNC-PP-0203-CualiAtlasti.pdf > Presentación.

Organización de las Naciones Unidas para la Educación la Ciencia y la Cultura - (UNEsCo). "Enfoque por competencias”. Oficina Internacional de Educación. Web. 7 Dic. 2011. http://www.ibe.unesco.org/es/comunidades/comunidad-de-practica-cop/ enfoque-por-competencias.html .
Páramo, Pablo. La investigación en las ciencias sociales - Técnicas de recolección de información. Bogotá: Universidad Piloto de Colombia, 2008. Impreso.

Pérez, Juan Gabriel. "Evaluación pedagógica de cursos virtuales”. VII Congreso Iberoamericano de Informática educativa. VII Congresso Iberoamericano de Informática Educativa, 2007. Universidad Complutense de Madrid. Web. 10 Feb. 2012. <http://www.niee.ufrgs.br/ eventos/RIBIE/2004/comunicacao/com374-383.pdf>

Salas, Julio. Estrategias para mejorar la comprensión lectora en el I ciclo de educación superior (Perú). Monografías.com. 2006. Web. 12 Feb. 2012. < http://www. monografias.com/trabajos44/comprension-lectora/ comprension-lectora2.shtml>

Siemens, George. "Connectivism: A Learning Theory for the Digital Age." International journal of Instructional Technology \& Distance Learning 2.1 (2005): 1-8. Web. 15 Feb. 2012. <http://er.dut.ac.za/bitstream/ handle/123456789/69/ Siemens_2005_Connectivism_A_learning_theory_for_the_digital_age.pdf?sequence $=1$ \&isAllowed $=y$ Web. Agosto 8 de 2011.>

Silvio, José. Reflexiones sobre la calidad en la educación virtual. Educoas. Web. 15 Jun. 2011. <http://www. educoas.org/portal/bdigital/lae-ducacion/139/pdfs/139pdf4.pdf>

Solano, Isabel. Las TIC como recursos en los procesos de comunicación didáctica. Tema 5: estrategias metodológicas. Digitum, Universiad de Murcia. 2010. Web. 15 Jun 2011. <https://digitum.um.es/xmlui/bitstream/10201/14622/1/ Tema5_estrategias\% 20metodol\%C3\%B3gicas_v2.pdf. Presentación>

Velandia, Crisanto. Modelo pedagógico con fundamento en cibernética social. Bogotá: Universidad Cooperativa de Colombia, 2005. Impreso.

---. Metodología interdisciplinaria. Universidad Cooperativa de Colombia. Bogotá: Siglo xxI, 2006. Impreso. 


\section{Apéndice A. Modelo de encuesta}

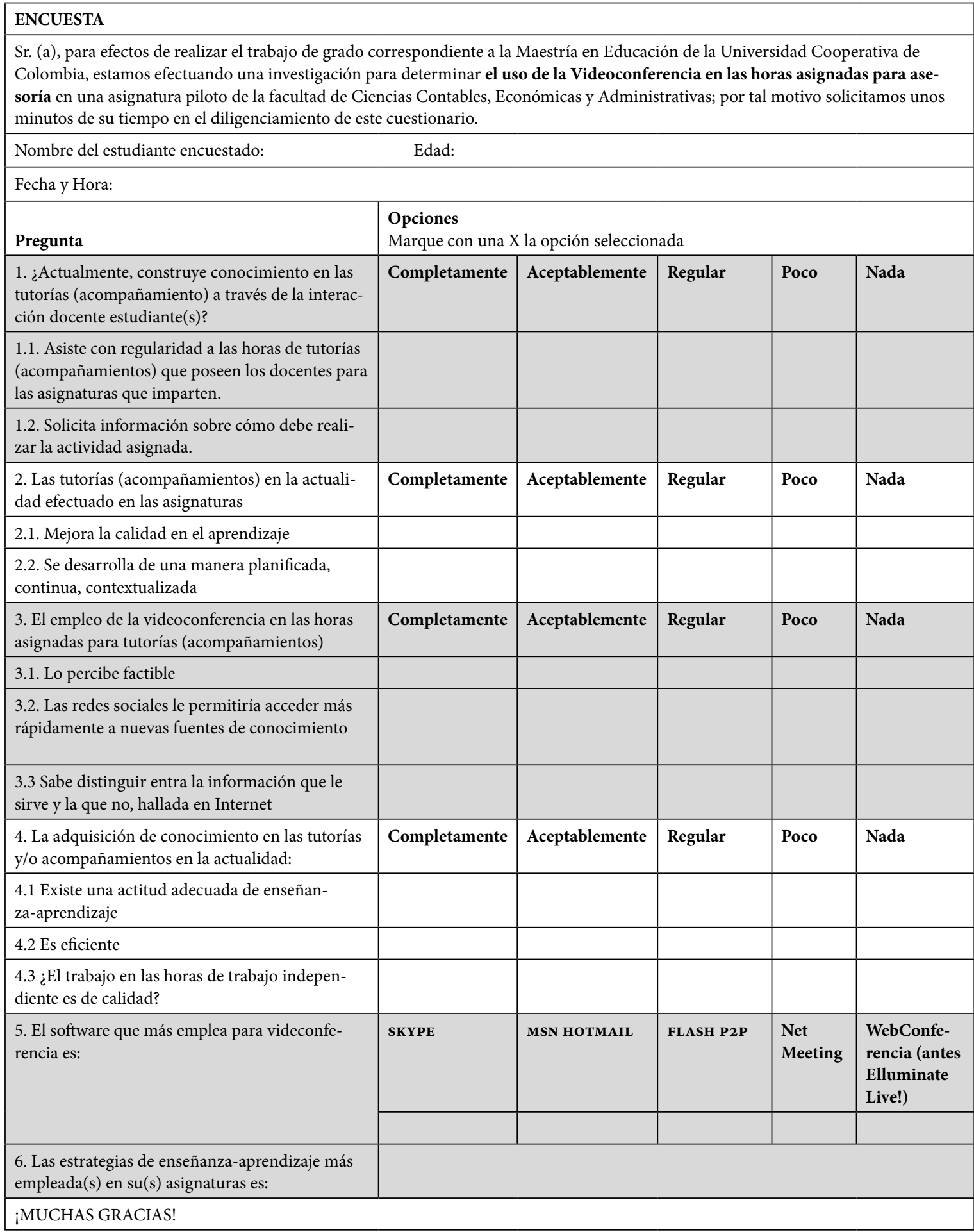

Fuente: elaboración propia. 


\section{Apéndice B. Paralelo resultados escala Likert entre docentes y estudiantes}

\begin{tabular}{|c|c|c|c|c|}
\hline \multirow[t]{2}{*}{ PREGUNTA } & \multicolumn{2}{|c|}{ PROFESORES } & \multicolumn{2}{|c|}{ ESTUDIANTES } \\
\hline & Promedio & Percepción & Promedio & Percepción \\
\hline $\begin{array}{l}\text { 1. ¿Actualmente, construye conocimiento en las tutorías (acompaña- } \\
\text { miento) a través de la interacción docente estudiante(s)? }\end{array}$ & 4,2 & Aceptable & 3,5 & Regular \\
\hline $\begin{array}{l}\text { 1.1. Asiste con regularidad a las horas de tutorías (acompañamientos) } \\
\text { que poseen los docentes para las asignaturas que imparten. }\end{array}$ & 3,4 & Regular & 4,1 & Regular \\
\hline $\begin{array}{l}\text { 1.2. Solicita información sobre cómo debe realizar la actividad asig- } \\
\text { nada. }\end{array}$ & 4,2 & $\begin{array}{l}\text { Cercanamente } \\
\text { Aceptable }\end{array}$ & 3,7 & $\begin{array}{l}\text { Cercanamente } \\
\text { Aceptable }\end{array}$ \\
\hline \multicolumn{5}{|l|}{$\begin{array}{l}\text { 2. Las tutorías (acompañamientos) en la actualidad efectuado en las } \\
\text { asignaturas }\end{array}$} \\
\hline 2.1. Mejora la calidad en el aprendizaje & 4,1 & Aceptable & 4,1 & Aceptable \\
\hline 2.2. Se desarrolla de una manera planificada, continua, contextualizada & 4,2 & Aceptable & 3,8 & $\begin{array}{l}\text { Cercanamente } \\
\text { Aceptable }\end{array}$ \\
\hline \multicolumn{5}{|l|}{$\begin{array}{l}\text { 3. El empleo de la videoconferencia en las horas asignadas para tutorías } \\
\text { (acompañamientos) }\end{array}$} \\
\hline 3.1. Lo percibe factible & 4,4 & Aceptable & 4,3 & Aceptable \\
\hline $\begin{array}{l}\text { 3.2. Las redes sociales le permitiría acceder más rápidamente a nuevas } \\
\text { fuentes de conocimiento }\end{array}$ & 4 & Aceptable & 4,3 & Aceptable \\
\hline $\begin{array}{l}\text { 3.3 Sabe distinguir entra la información que le sirve y la que no, hallada } \\
\text { en Internet }\end{array}$ & 4 & Aceptable & 4,4 & Aceptable \\
\hline \multicolumn{5}{|l|}{$\begin{array}{l}\text { 4. La adquisición de conocimiento en las tutorías y/o acompañamientos } \\
\text { en la actualidad: }\end{array}$} \\
\hline 4.1 Existe una actitud adecuada de enseñanza-aprendizaje & 4,2 & Aceptable & 4,1 & Aceptable \\
\hline 4.2 Es eficiente & 4,2 & Aceptable & 3,9 & $\begin{array}{l}\text { Cercanamente } \\
\text { Aceptable }\end{array}$ \\
\hline $4.3{ }_{¿} \mathrm{El}$ trabajo en las horas de trabajo independiente es de calidad? & 4,1 & Aceptable & 4,1 & Aceptable \\
\hline
\end{tabular}

Fuente: elaboración propia. 